1991;13:34-46.

3. Bryan CS. Clinical implications of positive blood cultures. Clin Microbiol Rev 1989;2:329-353.

4. Uzun Ö, Akalin E, Hayran M, Ünal S. Factors influencing prognosis in bacteremia due to gram-negative organisms: evaluation of 448 episodes in a Turkish university hospital. Clin Infect Dis 1992;15:866-873.

5. Setia U, Gross PA. Bacteremia in a community hospital. Arch Intern Med 1977;137:1698-1701.

6. Weinstein MP, Murphy JR, Reller LB, Lichtenstein KA. The clinical significance of positive blood cultures: a comprehensive analysis of 500 episodes of bacteremia and fungemia in adults, II: clinical observations, with special reference to factors influencing prognosis. Rev Infect Dis 1983;5:54-70.

7. Martin MA. Epidemiology and clinical impact of gram-negative sepsis. Infect Dis Clin North Am 1991;5:739-752.

8. Hilf M, Yu VL, Sharp J, Zuravleff JJ, Korvick JA, Muder R. Antibiotic therapy for Pseudomonas aeruginosa in a prospective study of 200 patients. Am J Med 1989;87:540-546.

9. Maki D, Schuna AA. A study of antimicrobial misuse in a university hospital. Am J Med Sci 1978;275:271-282.

10. Dunagan WC, Woodward RS, Medoff G, et al. Antimicrobial misuse in patients with positive blood cultures. Am J Med $1989 ; 87: 253-259$.

\title{
VRE and Long-Term Care
}

\section{by Gina Pugliese, RN, MS Martin S. Favero, PhD}

To assess the prevalence of VRE colonization among long-term-care facility (LTFC) residents requiring hospitalization, researchers studied colonization among admissions from 13 LTCFs to a private teaching hospital in the Chicago area. Rectal swab cultures were obtained from LTCF patients within 72 hours of admission to two medical wards and again at discharge. Samples were plated onto enterococcosel agar with $6 \mu \mathrm{g} / \mathrm{mL}$ of vancomycin. Molecular relatedness of the isolates was determined by pulsedfield gel electrophoresis; strains were defined by $>6$ band differences.

Overall, 19 (63\%) of 30 LTCF patients were found to be colonized with vancomyci-resistant enterococci (VRE) during this hospitalization.
Ten strains were identified-2 Enterococcus faecalis, 5 Enterococcus faecium, 2 Enterococcus gallinarum, and 1 Enterococcus avium. Fourteen (47\%) of 30 of the patients harbored VRE on admission (8 strains). Five of the 16 VRE-negative patients acquired VRE by discharge (4 strains). Five positive patients acquired a second VRE strain. LTCF patients maintained a VRE prevalence of twice that of other ward patients during this time. VRE-positive admissions came from 9 of 13 LTCF represented. Histories of antibiotic use, hospitalization within the prior 60 days, and presence of decubitus ulcers were associated strongly with VRE colonization on admission. Only one LTCF patient was clinically infected with VRE (hemodialysis graft bacteremia).

The researchers concluded that almost half of LTCF patients requiring hospitalization carried VRE at admission; $31 \%$ of negative LTCF patients acquired VRE in the hospital. LTCF patients have become a reservoir for multiple strains of VRE and may transfer VRE to and from acutecare hospitals. Presumptive isolation and cohorting of LTCF patients at highest risk for VRE colonizationthose with skin breakdown, recent hospitalization, or antibiotic exposure-should be studied in hospitals and LTCF.

FROM: Elizaga ML, Beezhold D, Hayden MK, et al. The prevalence and colonization with vancomycin-resistant enterococci (VRE) among hospitalized residents of long-term-care facilities. Presented at the 34th Annual Meeting of the Infectious Disease Society of America; September 18-20, 1996; New Orleans, LA. Abstract 352. 